\title{
The Effects of the Health System Response to the COVID-19 Pandemic on Chronic Disease Management: A Narrative Review
}

This article was published in the following Dove Press journal: Risk Management and Healthcare Policy

\author{
Tetyana Kendzerska (D) ${ }^{1-3}$ \\ David T Zhu ${ }^{1,4}$ \\ Andrea S Gershon iD $^{5}$ \\ Jodi D Edwards ${ }^{3,6}$ \\ Cayden Peixoto ${ }^{7}$ \\ Rebecca Robillard (1D ${ }^{8}$ \\ Claire E Kendall $1,7,9,10$
}

'Clinical Epidemiology Program, The Ottawa Hospital Research Institute/The Ottawa Hospital, Ottawa, Ontario, Canada; ${ }^{2}$ Department of Medicine, University of Ottawa, Ottawa, Ontario, Canada; ${ }^{3}$ School of Epidemiology and Public Health, University of Ottawa, Ottawa, Ontario, Canada; ${ }^{4}$ Faculty of Science, Western University, London, Ontario, Canada; ${ }^{5}$ Department of Medicine, Sunnybrook Health Sciences Centre/University of Toronto, Toronto, Ontario, Canada; ${ }^{6}$ University of Ottawa Heart Institute, Ottawa, Ontario, Canada; ${ }^{7}$ The Institut Du Savoir

Montfort, Ottawa, Ontario, Canada; ${ }^{8}$ The Royal's Institute of Mental Health Research/University of Ottawa, Ottawa, Ontario, Canada; ${ }^{9}$ C.T. Lamont Primary Health Care Research Group, Bruyère Research Institute, Ottawa, Ontario, Canada; ${ }^{10}$ Department of Family Medicine, University of Ottawa, Ottawa, Ontario, Canada
Correspondence: Tetyana Kendzerska The Ottawa Hospital Research Institute, 50I Smyth Road, Ottawa, Ontario KIH 8L6, Canada

$\mathrm{Tel}+\mid$ 6|3-76|-4636

Email tkendzerska@toh.ca
Background: Individuals with chronic conditions require ongoing disease management to reduce risks of adverse health outcomes. During the COVID-19 pandemic, health care for non-COVID-19 cases was affected due to the reallocation of resources towards urgent care for COVID-19 patients, resulting in inadequate ongoing care for chronic conditions.

Methods: A keyword search was conducted in PubMed, Google Scholar, Science Direct, and Scopus for English language articles published between January 2020 and January 2021.

Findings: During the COVID-19 pandemic, in-person care for individuals with chronic conditions have decreased due to government restriction of elective and non-urgent healthcare visits, greater instilled fear over potential COVID-19 exposure during in-person visits, and higher utilization rates of telemedicine compared to the pre-COVID-19 period. Potential benefits of a virtual-care framework during the pandemic include more effective routine disease monitoring, improved patient satisfaction, and increased treatment compliance and follow-up rates. However, more needs to be done to ensure timely and effective access to telemedicine, particularly for individuals with lower digital literacy. Capitation primary care models have been proposed as a more financially-robust approach during the COVID-19 pandemic than fee-for-service primary care models; however, the interplay between different primary models and the health outcomes is still poorly understood and warrants further investigation. Shortages of medication used to manage chronic conditions were also observed at the beginning of the COVID-19 pandemic due to global supply chain disruptions. Finally, patients with chronic conditions faced lifestyle disruptions due to the COVID-19 pandemic, specifically in physical activity, sleep, stress, and mental health, which need to be better addressed.

Interpretation: Overall, this review elucidates the disproportionately greater barriers to primary and specialty care that patients with chronic diseases face during the COVID-19 pandemic and emphasizes the urgent need for better chronic disease management strategies moving forward.

Keywords: narrative review, chronic disease, COVID-19 pandemic, primary care, specialist care

\section{Introduction}

Chronic diseases represent the leading cause of disability worldwide, with approximately one in three adults suffering from one or more chronic conditions. ${ }^{1}$ Chronic diseases, with the most prevalent conditions being cardiovascular disease, chronic lung disease, diabetes, and chronic kidney disease, have been consistently associated with higher healthcare utilization and worse health outcomes when care is 
disrupted. ${ }^{1-3}$ The likelihood of hospital admission for an adverse event increases with the number of chronic conditions, especially in the elderly population. ${ }^{1}$ While the complex interplay of chronic disease management is seldom seamless under normal circumstances, the novel barriers imposed by the COVID-19 pandemic increase this difficulty substantially. Globally, the pandemic has strained healthcare systems' resources and adversely affected clinical decision-making by limiting ancillary testing (eg, bloodwork, imaging, pulmonary function tests) and physical exams. ${ }^{4-6}$ Further, in March 2020, governments around the world ubiquitously imposed restrictions on the use of hospital and ambulatory services by cancelling all elective, routine, and non-urgent patient procedures, enforcing stricter physical distancing measures, and transitioning into remote care to reallocate resources towards the urgent care of COVID-19 patients. ${ }^{7}$ This has hindered the ability of individuals with chronic conditions to receive timely and effective access to primary and specialty care. ${ }^{8-10}$ As the COVID19 pandemic progresses, there is still considerable uncertainty surrounding the management of individuals with chronic conditions. Thus, in this narrative review, we aim to overview how individuals with chronic conditions have been affected by changes in (1) adaptions in the healthcare system for both primary and specialty care, (2) levels of healthcare utilization (eg, hospitalizations, emergency department [ED] visits, use of ancillary testing, medications supply), and (3) socioeconomic and environmental risk factors, in response to the COVID-19 pandemic.

\section{Methods}

Between July 2020 and January 2021, we conducted a literature search in PubMed, Google Scholar, Science Direct, and Scopus for articles published between January 2020 and January 2021 (with an exception to this time range if the article was only used to contextualize general challenges and epidemiology of chronic disease patients). Combinations of the search terms "chronic disease", "COVID-19", "cardiovascular disease", "coronary artery disease", "heart failure", "myocardial infarction", "hypertension", "chronic obstructive pulmonary disease", "asthma”, "diabetes", “epilepsy”, "insomnia”, "obstructive sleep apnea", "telemedicine", "drug shortages", "diet", "nutrition", "physical exercise", "primary care", "hospitalization", "fee-for-service", "capitation", "mental health", and "stress" were used to initially retrieve articles. We screened titles and abstracts using the following criteria.
Inclusion criteria were defined as any English-language peer-reviewed primary articles, review articles, or opinion and commentary articles that focused on, or at least included, non-SARS-CoV-2 infected patients with chronic diseases during the COVID-19 pandemic. Exclusion criteria were defined as any sources that did not fit into these criteria, such as articles that focused only on SARS-CoV-2 infected patients with comorbid chronic diseases. Articles that met inclusion criteria were subsequently evaluated in their entirety for content relevancy to our narrative discussion. All authors agreed on the final reference list.

\section{Healthcare Characteristics and Utilization by Individuals with Chronic Conditions During the COVID-I 9 Pandemic Primary Care Characteristics and Utilization by Individuals with Chronic Conditions}

Primary care serves as a gateway into the healthcare system and promotes better health outcomes for individuals with chronic conditions through monitoring, management, and follow-up. To mitigate the spread of COVID-19 infection, primary care centres around the world concertedly transitioned into remote consultations with ad hoc inperson medical visits. Commonly, this is presented as a three-step framework: (1) patients are triaged remotely and reviewed for travel history, contact history, and COVID-19 symptoms; (2) patients with chronic diseases or mild COVID-19 symptoms are advised to quarantine at home while routinely consulting with physicians remotely by video, phone, email, etc.; (3) patients with substantially-worsened chronic diseases or severe COVID-19 symptoms who require urgent care are hospitalized. ${ }^{11-15}$ In theory, this framework optimizes the distribution of personal protective equipment (PPE) and staff to urgently treat individuals with COVID-19 and protects patients with chronic diseases from potential COVID-19 exposure in hospitals and clinics. However, in practice, the efficacy of such measures is largely dictated by the availability of financial and infrastructural resources. A recent crosssectional study found that within resource-rich settings and consistent access to a regular primary care physician prior to the pandemic, in-person outpatient visits for chronic conditions fell by $60 \%$ to $70 \%$, while there was a $60 \%$ to $100 \%$ increase in video and telephone 
consultations. ${ }^{16}$ This smooth shift into remote care resulted in higher patient satisfaction and consistent noshow rates than before the onset of COVID-19, reflecting these patients continued timely and effective access to primary care during the COVID-19 pandemic. $^{16,17}$ However, even in resource-rich settings, there is a high degree of variability among different communities; ${ }^{18}$ specifically, inequalities in chronic diseases and the social determinants of health among minority ethnic groups, people living in areas of higher socioeconomic deprivation, individuals living in poverty, and other marginalised groups. ${ }^{19}$ Further, resource-constrained and low-income settings may force individuals with chronic conditions to either continue in-person medical visits, risking potential COVID-19 exposure or stay at home and seek medical care only after their conditions have substantially worsened. This stretches existing health inequities for socioeconomically-disadvantaged patients with chronic diseases and has impeded the management of their diseases; thus, worsening health outcomes. ${ }^{20}$ For example, among people with diabetes in such settings, $80 \%$ were concerned with their ability to ensure timely follow-up with clinicians, and more than $70 \%$ reported worsening of glycemic control manifesting as higher fasting blood glucose levels, mainly due to their inability to reach primary care clinicians for modulating insulin dosages. ${ }^{21,22}$

Interestingly, these reductions in face-to-face primary care visits by patients with chronic conditions have substantial financial consequences for healthcare systems. A large-scale modelling study of US outpatient and primary care centres estimated that a conservative $6 \%$ reduction in total visit volume leads to a nearly $\$ 70,000$ (USD) loss per full-time physician and a net $\$ 15$ billion (USD) loss across all US primary care centres. ${ }^{23}$ Further, for over half of these clinicians who rely on independent small practices and likely have less capital to smoothly transition into telemedicine, these financial losses could balloon twofold, (1) risking the closure of their practices or staff layoffs, and (2) suggesting incompatibility of patient-centered care with fee-for-service (FFS) payment. ${ }^{24,25}$ Weakened primary care systems are likely to adversely impact the quality of care of individuals with chronic conditions for years beyond the COVID-19 pandemic. $^{23}$ In Ontario (Canada), a survey of 4800 fee-for-service primary care physicians found that nearly $50 \%$ had already laid off staff and may need to close their practices unless they receive additional financial support. ${ }^{26}$ Early evidence suggests that capitation primary care models, which are not reliant on the volume of services but instead on delivering costeffective care for a specified patient volume, maybe bettersuited to ensure the robustness of primary care during COVID-19. ${ }^{24}$ Capitation primary care physicians may be incentivized to ensure that patients remain healthy to limit any future visits and subsequent care needed. ${ }^{27-29}$ It has been previously shown that compared to fee-for-service primary care models, capitation may yield higher rates of patients receiving recommended management and favourable health outcomes, ${ }^{30,31}$ while also reducing admission rates for individuals with chronic conditions. ${ }^{32}$ A reduction in hospitalization rates due to primary care improvement is especially critical during the COVID-19 pandemic to free resources for the urgent care of individuals with COVID-19. Despite benefits offered by capitation models, a large concern is that capitation primary care providers may be financially-incentivized to avoid enrolling or undertreat patients with chronic conditions, as these patients are associated with recurrent admissions (both virtual and in-person); hence, capitation rates may need to be adjusted for patients with chronic conditions to reflect the higher expected costs. ${ }^{33}$ Further, the efficacy of different primary care models for patients with chronic conditions during the COVID-19 pandemic remains poorly understood; thus, more research is urgently needed contrasting the efficacy and access to care of capitation models compared to FFS models for patients with chronic conditions, specifically during the COVID-19 pandemic, as well as potential areas of risks for both models.

\section{Reductions in Chronic Disease-Related ED Visits and Hospitalizations}

The COVID-19 pandemic also led to significant reductions in hospitalizations, ED visits, and inpatient visits for non-COVID-19 patients with chronic conditions, notably, during the period of March 2020 to April 2020. Individuals with acute myocardial infarction, stroke, heart failure, and other chronic cardiovascular conditions experienced a significant ( 40\%) decrease in hospital admissions and ED visits during the COVID-19 pandemic compared to baseline. ${ }^{34-39}$ Rates of heart failure-related hospitalizations decreased $40-50 \%$ during the COVID-19 period compared to before $;{ }^{34,40}$ however, one study found a three-fold increase in mortality related to heart-failure. ${ }^{41}$ Overall, these trends are concerning, suggesting that patients with chronic cardiovascular conditions tend to avoid in- 
person medical visits to hospitals, clinics, and ED possibly due to fear of potential COVID-19 exposure. In a cross-sectional study of 185,000 North American patients, one-third reported cancelling pre-scheduled appointments, and over $40 \%$ reported elevated worry regarding in-person medical visits. ${ }^{42}$ Hypertension and cardiovascular conditions have often been shown as the two leading comorbidities in COVID-19 related mortality, raising the risk two-fold. Therefore, chronic cardiac patients may be especially prudent about minimizing inperson healthcare visits during the COVID-19 pandemic. ${ }^{43-46}$ Especially vulnerable are elderly patients with chronic conditions. Elderly individuals are at greater susceptibility to COVID-19 infection, worse prognosis following COVID-19 infection, and are also more likely to have one or more non-communicable diseases; thus, they may face heightened levels of instilled fear of COVID-19 exposure during in-person medical visits. ${ }^{47}$ Further, elderly patients with chronic diseases have a higher prevalence of moderate to severe dementia $^{48,49}$ and cognitive impairment, which may limit their ability to effectively manage their chronic conditions. People with dementia also face worse health outcomes following COVID-19 exposure, including a nearly 3-fold elevated risk of mortality. ${ }^{50}$ Altogether, elderly patients are a subset of the chronic disease population that may face especially amplified fear and difficulty maintaining chronic disease healthcare routines due to the COVID-19 pandemic.

Two alternative theories have been proposed to explain reductions in chronic disease-related ED visits and hospitalizations in addition to possible fear of potential COVID-19 exposure due to in-person medical visits. First, the reduction in hospitalizations may also be attributed to the mitigation of other lifestyle and environmental risk factors during quarantine, such as reduced exposure to traffic, air pollution, other respiratory infections (eg, influenza), as well as reduced energy expenditure. ${ }^{35,51}$ This is supported by a decline in hospitalizations and ED visits for chronic obstructive pulmonary disease (COPD)/asthma and pneumonia during the COVID-19 pandemic, which shares many of these risk factors. ${ }^{37,52,53}$ Another possible explanation is that the effective shift into telemedicine, coupled with the absence of prior distractions (eg, work, travel, etc.), has led to increased treatment adherence and improved management of chronic conditions. ${ }^{54,55}$

\section{Deployment of Telemedicine for Chronic Disease Management}

Prior to the COVID-19 outbreak, telemedicine had already been distinguished as an effective strategy to monitor and manage chronic illnesses, for example, for patients with heart failure and COPD. ${ }^{56,57}$ Suddenly, social distancing, quarantine, and hospital restriction measures have forced individuals with chronic conditions to re-envision their routine treatments remotely with ad hoc in-person medical visits. This has led to widespread and significant increases in telemedicine utilization. ${ }^{58-60}$ A rapid review found that telemedicine utilization had increased by nearly $90 \%$ in the US during the COVID-19 pandemic. ${ }^{61}$ Common uses of telemedicine include video or phone consultations, allowing individuals with chronic conditions to selfmonitor symptoms and send this information to their clinicians, mobile apps, and platforms. ${ }^{55,62}$ For chronic heart failure patients, virtual care has enabled effective routine monitoring (eg, heart rhythm, blood pressure, $\mathrm{O}_{2}$ saturation) associated with better compliance with treatment rates $(51 \%$ vs $35 \%)$ and follow-up rates than in-person care during COVID-19. ${ }^{63}$ Among patients with chronic respiratory conditions such as asthma or COPD, remote care has been effective for self-management education (eg, inhaler device training, breathing techniques, recognizing symptoms, forming or complying with action plans, etc.), leading to improved health outcomes. ${ }^{52,64}$ For people with epilepsy, virtual services like electronic seizure journals, mobile apps, messaging, and video conferencing have led to high patient satisfaction rates, lower no show rates, and better health outcomes. ${ }^{65}$

Despite these advantages, telemedicine is associated with many barriers to implementation, including (1) limited ability to perform physical exams or ancillary testing, (2) reduced ability to pick up verbal and nonverbal cues, which could result in more diagnostic errors, and (3) a lack of access to technology and limitations inherent to variations in digital literacy, especially in elderly patients for whom chronic conditions are common. ${ }^{64-66}$ Digital illiteracy is more common among socioeconomically disadvantaged, racialized, older, and rural-residing individuals, which may contribute to lower telemedicine access by these populations. Consistent with this, rates of telemedicine visits for chronic disease patients have been found to be reduced among those who have lower-income or older age ( $>60$ years), are racialized (Hispanic, Black, Asian/ Pacific Islander), living in rural areas, and non-English 
speaking. ${ }^{67,68}$ Moreover, previous research has found that among the one in eight Americans who live in poverty, the proportion who own smartphones, have broadband access to the internet, and have basic digital literacy are $30 \%$, $40 \%$, and $50 \%$ lower, respectively, than those not living in poverty. ${ }^{69}$ These findings highlight a public health need to increase the financial and technological accessibility of telemedicine services, especially in the context of the COVID-19 pandemic. Therefore, unless telemedicine services address the above disparities, pre-existing health inequities will be further exacerbated. ${ }^{70}$ Ultimately, more needs to be done to provide patients with chronic diseases, especially those from socioeconomically disadvantaged groups, with technological and financial resources, such as broadband internet access and digital devices, as well as support to develop digital skills. ${ }^{67}$

\section{Shortages of Medication Supply for Individuals with Chronic Diseases}

During the COVID-19 pandemic, medications supplies have fluctuated due to the global disruption of supply and transportation chains, manufacturing restrictions due to insufficient raw materials or social distancing measures for workers, stockpiling and panic-buying behaviour in some patients, and higher demands to treat or develop clinical trials for COVID-19 patients, which has caused medicine shortages for patients with chronic conditions. $^{71-73}$ Notably, hydroxychloroquine, which is used for treating chronic autoimmune diseases like lupus and rheumatoid arthritis, faced tripled demand during COVID-19 as a promising therapeutic medication for treating patients with COVID-19, leading to major shortages. $^{74}$ At the early outbreak period, medications like dapagliflozin and metformin used to treat diabetes, ${ }^{75}$ and midazolam and propofol, which are sedatives that have been used to treat a chronic respiratory condition such as COPD, ${ }^{76}$ faced critical shortages due to demand in treating COVID-19 patients on mechanical ventilation. Among COPD and asthma patients, concerns surrounding the use of nebulizers and non-invasive ventilation in aerosolizing the COVID-19 virus, thus risking exposure to healthcare workers, have substantially increased demand for metered-dose inhalers and subsequently inhaler shortages. ${ }^{77}$ Medicine shortages will likely lead to treatment interruption and worsening of chronic conditions symptoms if they continue. However, there is still limited evidence of their direct downstream effects on short- and long-term health outcomes in patients with chronic diseases.

\section{Changes in Lifestyle and Related Risk Factors for Individuals with Chronic Conditions \\ Barriers to Physical Activity and Exercise for Patients with Chronic Diseases During COVID-19}

As aforementioned, social distancing and quarantine measures have mitigated the spread of COVID-19. However, this also has collaterally limited opportunities for individuals with chronic conditions to participate in structured exercised programs (eg, group fitness sessions, team sports) as well as in-person physiotherapy programs specific to their conditions. ${ }^{78-80}$ Alternative exercise strategies have emerged, such as social media, virtual support groups, and mobile fitness apps, which provide a motivating and social environment, helping individuals with chronic conditions attain their physical activity goals and notably prevent obesity. ${ }^{81,82}$ Studies found that during the COVID-19 pandemic physical activity and exercise declined by approximately $40 \%$ in the general population. ${ }^{83,84}$ Similarly, cross-sectional studies reported that moderate- and high-intensity daily physical activity were decreased by 30 and 11 minutes, respectively, with $70 \%$ of participants, including individuals with chronic conditions, reporting overall less physical activity. ${ }^{78,80}$ Obese patients with chronic diseases are at aggravated risk for cardiovascular, metabolic, and respiratory complications; thus, leading to worse health outcomes for their pre-existing conditions, ${ }^{79}$ which may result in an even greater instilled fear of potential COVID-19 exposure during in-person medical visits. ${ }^{85}$ Altogether, it is essential that individuals with chronic conditions ensure adequate levels of regular physical activity during the COVID-19 pandemic to prevent exacerbations of their conditions and promote better health outcomes. ${ }^{86}$

\section{Psychosocial and Mental Health Considerations for Individuals with Chronic Conditions During COVID-19}

A higher prevalence of comorbid mental disorders such as depression, anxiety, and chronic stress has been previously found in individuals with chronic physical conditions. ${ }^{1-3,87}$ They consistently report more social isolation, uncertainty 
about the future, and guilt stemming from the actual or perceived physical and psychosocial limitations of their disease. These three outcomes, which may likely be worsened by the COVID-19 pandemic, can exacerbate the morbidity of their chronic disease, creating a vicious cycle. ${ }^{87,88}$ For individuals with chronic cardiovascular diseases, greater stress levels have been previously documented as a risk factor for atherosclerosis, development of hypertension, and recurrent coronary heart disease, ${ }^{89-91}$ particularly with a $40-50 \%$ elevated incidence of recurrent coronary heart disease. ${ }^{91}$ A US cross-sectional study found that, compared to the general population, patients with chronic conditions experience higher stress levels due to being at higher risk for worse health outcomes from COVID-19 infection. ${ }^{92}$ For instance, among patients with epilepsy, elevated stress levels and mental health issues were highly prevalent ${ }^{93,94}$ with higher seizure frequency during the COVID-19 outbreak, which is consistent with the amplified worry of being unable to receive timely and effective access to primary care and antiseizure medications. ${ }^{95}$ Correspondingly, in one study, $73 \%$ and $40 \%$ of patients with epilepsy reported that obtaining routine anti-epilepsy medications and managing stress levels, respectively, were made "more difficult" by the COVID-19 pandemic through a wide array of factors (greater difficulty with reaching their epilepsy healthcare providers, difficulty with maintaining normal sleep routines, greater difficulty with obtaining anti-epilepsy medications and with routinely taking these medications). ${ }^{87}$ Notably, one-third of patients in this study reported increased seizure frequency during the COVID-19 pandemic. $^{95}$

\section{Sleep Disruption and Disorders for Individuals with Chronic Conditions During COVID-I9}

The COVID-19 pandemic significantly amplified the frequency and severity of sleep disruptions. Notably, occurrences of clinically meaningful sleep difficulties have increased from $30 \%$ to $40 \%$ since the COVID-19 pandemic among the general population. ${ }^{96-98}$ Moreover, this increase has been nearly two-fold among individuals with chronic conditions ${ }^{99,100}$ which may be explained by higher rates of mental illness and stress during the COVID-19 pandemic, which could provoke cognitive arousal and lead to sleep disruptions. ${ }^{101}$ Additionally, social distancing and quarantine mandates have led to a greater proportion of individuals with chronic conditions feeling lonely or socially isolated, which has been bidirectionally associated with disrupted sleep patterns. ${ }^{102}$ Further, atypical daily routines, sleep-wake times, meal and activity patterns, and often reduced light exposure may have led to the dysregulation of circadian rhythms, levels of arousal, and sleep drive in patients with chronic diseases. ${ }^{103}$ Despite the disruptions, many jobs and activities have transitioned into being completed at-home or remotely, thus offering more flexible schedules, and subsequently, the opportunity for patients to re-align their regular sleep-wake schedules to better fit their chronotypes and to better adhere to chronic illness management strategies. ${ }^{101}$

Treatment with positive airway treatment devices (PAP) for individuals with sleep-disordered breathing can be challenging during the COVID-19 pandemic because of PAP-induced droplets and aerosol, which act as mediums to transmit or spread the virus (SARS-CoV-2) ${ }^{104}$ Despite this, among obstructive sleep apnea (OSA) patients, compliance with continuous positive airway pressure (CPAP) therapy increased by $27 \%$ during the quarantine. ${ }^{105}$

Sleep health education must be prioritized as part of chronic disease management to promote better sleep quality and quantity for individuals with chronic conditions to prevent exacerbation of their conditions, which may be delivered through existing telemedicine channels. ${ }^{103}$

\section{Limitations}

This is a narrative review to broadly describe how individuals with chronic conditions have been affected by the health system response to the COVID-19 pandemic rather than a systematic review of a focused research question. Further, we only included articles published between July 2020 and January 2021; thus, due to the rapid rate of knowledge advancement and new publications on the management of patients with chronic diseases during the COVID-19 pandemic, this review only captures peerreviewed articles related to the early and middle stages of the pandemic. For example, at the time of writing, there was insufficient literature to illustrate how the introduction of SARS-CoV-2 vaccines will influence healthcare received by non-SARS-CoV-2 individuals with chronic conditions. Further research is warranted to review the continuously-evolving landscape of chronic disease management as the COVID-19 pandemic progresses in order to guide healthcare service delivery in the later stages of the pandemic, the post-pandemic phase, and the next outbreak. We also limited the number of 
databases searched to specific keywords, restricted to articles in English language, and restricted the types of literature. As more literature becomes available, researchers should conduct systematic reviews with focused questions pertaining to the care of individuals with chronic conditions during the COVID-19 pandemic and should broaden the search strategy to include all potentially relevant literature, such as grey literature and other non-peerreviewed sources.

\section{Conclusion}

The purpose of this narrative review was to provide an overview of how individuals with chronic conditions have been affected by changes in adaptions in the healthcare system, healthcare utilization, and socioeconomic and environmental risk factors in response to the COVID-19 pandemic. During the COVID-19 pandemic, in-person primary and specialty care for individuals with chronic conditions have substantially decreased due to government restriction of elective and non-urgent healthcare visits, as well as greater instilled fear over potential COVID-19 exposure during in-person visits. Utilization rates of telemedicine increased compared to the preCOVID-19 period. Potential benefits of a virtual-care framework during the pandemic include more effective routine disease monitoring, improved patient satisfaction, and increased treatment compliance and follow-up rates. However, more needs to be done on the part of healthcare systems to ensure timely and effective access to telemedicine, particularly for individuals with lower digital literacy, notably in people with lower socioeconomic status. Additionally, capitation primary care models have been proposed as a more financially-robust approach during the COVID-19 pandemic than fee-for-service primary care models. Furthermore, shortages of medications used to manage chronic conditions have occurred (more notably at the beginning of the pandemic) due to global supply chain disruptions further affecting chronic disease management.

Individuals with chronic conditions have also faced significant lifestyle disruptions due to the COVID-19 pandemic, particularly regarding physical activity, sleep, stress, and mental health, which need to be better addressed by healthcare systems in the COVID-19specific context. Conversely, mitigation of some lifestyle and environmental risk factors during quarantine, such as reduced exposure to traffic, air pollution, and other respiratory infections, may improve outcomes in individuals with chronic conditions.

The information summarized and provided by this review can be used as a foundation for further research studies and to guide healthcare service delivery in later stages of the pandemic, the post-pandemic phase, and during the next outbreak.

\section{Data Sharing Statement}

Data sharing does not apply to this article as no datasets were generated or analyzed during the current study.

\section{Author Contributions}

All authors made substantial contributions to conception and design, acquisition of data, or analysis and interpretation of data; took part in drafting the article or revising it critically for important intellectual content; agreed to submit to the current journal; gave final approval of the version to be published; and agree to be accountable for all aspects of the work.

\section{Funding}

The Ottawa Hospital Academic Medical Organization (TOHAMO).

\section{Disclosure}

TK is supported by the PSI foundation (the 2020 PSI Graham Farquharson Knowledge Translation Fellowship). The other authors declare that they have no competing interests.

\section{References}

1. Hajat C, Stein E. The global burden of multiple chronic conditions: a narrative review. Prev Med Rep. 2018;12:284-293.

2. Roberts KC, Rao DP, Bennett TL, Loukine L, Jayaraman GC. Prevalence and patterns of chronic disease multimorbidity and associated determinants in Canada. Health Promot Chronic Dis Prev Can. 2015;35(6):87-94.

3. Cohen SP, Baber ZB, Buvanendran A, et al. Pain management best practices from multispecialty organizations during the COVID-19 pandemic and public health crises. Pain Med. 2020;21(7):1331-1346.

4. Laupacis A. Working together to contain and manage COVID-19. CMAJ. 2020;192(13):E340-E341.

5. Williams S, Tsiligianni I. COVID-19 poses novel challenges for global primary care. NPJ Prim Care Respir Med. 2020;30(1):30.

6. Kouri A, Gupta S, Yadollahi A, et al. Addressing reduced laboratory-based pulmonary function testing during a pandemic. Chest. 2020;158(6):2502-2510.

7. Eccleston C, Blyth FM, Dear BF, et al. Managing patients with chronic pain during the COVID-19 outbreak. considerations for the rapid introduction of remotely supported (eHealth) pain management services. Pain. 2020;161(5):889-893. 
8. Weinstein E, Ragazzoni L, Burkle F, Allen M, Hogan D, Della Corte F. Delayed primary and specialty care: the coronavirus disease-2019 pandemic second wave. Disaster Med Public Health Prep. 2020;14(3):e19-e21.

9. Deer TR, Sayed D, Pope JE, et al. Emergence From the COVID-19 pandemic and the care of chronic pain: guidance for the Interventionalist. Anesth Analg. 2020;131(2):387-394.

10. The Lancet Respiratory M. COVID-19 heralds a new era for chronic diseases in primary care. Lancet Respir Med. 2020;8 (7):647.

11. Greenhalgh T, Koh GCH, Car J. Covid-19: a remote assessment in primary care. BMJ. 2020;368:m1182.

12. Razai MS, Doerholt K, Ladhani S, Oakeshott P. Coronavirus disease 2019 (covid-19): a guide for UK GPs. BMJ. 2020;368: $\mathrm{m} 800$.

13. Chang BB, Chiu TY. Ready for a long fight against the COVID-19 outbreak: an innovative model of tiered primary health care in Taiwan. BJGP Open. 2020;4:2.

14. Morreel S, Philips H, Verhoeven V. Organisation and characteristics of out-of-hours primary care during a COVID-19 outbreak: a real-time observational study. PLoS One. 2020;15(8):e237629.

15. Lim WH, Wong WM. covid-19: notes from the front line, singapore's primary health care perspective. Ann Fam Med. 2020;18 (3):259-261

16. Joy M, McGagh D, Jones N, et al. Reorganisation of primary care for older adults during COVID-19: a cross-sectional database study in the UK. Br J Gen Pract. 2020;70(697):e540-e547.

17. Majeed A, Maile EJ, Bindman AB. The primary care response to COVID-19 in England's National Health Service. $J R$ Soc Med. 2020;113(6):208-210.

18. Ahmed S, Shommu NS, Rumana N, Barron GR, Wicklum S, Turin TC. Barriers to access of primary healthcare by immigrant populations in canada: a literature review. J Immigr Minor Health. 2016;18(6):1522-1540.

19. Bambra C, Riordan R, Ford J. The COVID-19 pandemic and health inequalities. J Epidemiol Community Health. 2020;74 (11):964-968.

20. Garg S, Basu S, Rustagi R, Borle A. Primary health care facility preparedness for outpatient service provision during the covid-19 pandemic in india: cross-sectional study. JMIR Public Health Surveill. 2020;6(2):e19927.

21. Pal R, Yadav U, Verma A, Bhadada SK. Awareness regarding COVID-19 and problems being faced by young adults with type 1 diabetes mellitus amid nationwide lockdown in India: a qualitative interview study. Prim Care Diabetes. 2020.

22. Banerjee M, Chakraborty S, Pal R. Diabetes self-management amid COVID-19 pandemic. Diabetes Metab Syndr. 2020;14 (4):351-354

23. Basu S, Phillips RS, Phillips R, Peterson LE, Landon BE. Primary care practice finances in the united states amid the COVID-19 Pandemic. Health Aff. 2020;39(9):1605-1614.

24. Tao W, Agerholm J, Burstrom B. The impact of reimbursement systems on equity in access and quality of primary care: a systematic literature review. BMC Health Serv Res. 2016;16(1):542.

25. DeVoe JE. The incompatibility of patient-centered care with fee-for-service payment. JAMA Intern Med. 2020.

26. Glauser W. Pandemic amplifies calls for alternative payment models. CMAJ. 2020;192(23):E638-E639.

27. Li X, Krumholz HM, Yip W, et al. Quality of primary health care in China: challenges and recommendations. Lancet. 2020;395 (10239):1802-1812.

28. Fuchs VR. Health Care Policy After the COVID-19 Pandemic. JAMA. 2020;324(3):233-234.

29. Liao JM. COVID-19 Recovery Will Involve Strategy, Not Just Operational Effectiveness. J Am Coll Radiol. 2020;17(10): 1334-1336.
30. Tu K, Cauch-Dudek K, Chen Z. Comparison of primary care physician payment models in the management of hypertension. Can Fam Physician. 2009;55(7):719-727.

31. Kiran T, Kopp A, Moineddin R, Glazier RH. Longitudinal evaluation of physician payment reform and team-based care for chronic disease management and prevention. CMAJ. 2015;187 (17):E494-E502.

32. Liddy C, Singh J, Hogg W, Dahrouge S, Taljaard M. Comparison of primary care models in the prevention of cardiovascular disease - a cross sectional study. BMC Fam Pract. 2011;12:114.

33. Grasso ME, Weller WE, Shaffer TJ, Diette GB, Anderson GF. Capitation, managed care, and chronic obstructive pulmonary disease. Am J Respir Crit Care Med. 1998;158(1):133-138.

34. Frankfurter C, Buchan TA, Kobulnik J, et al. Reduced Rate of Hospital Presentations for Heart Failure During the COVID-19 Pandemic in Toronto, Canada. Can J Cardiol. 2020;36(10): $1680-1684$.

35. Papafaklis MI, Katsouras CS, Tsigkas G, et al. "Missing" acute coronary syndrome hospitalizations during the COVID-19 era in Greece: medical care avoidance combined with a true reduction in incidence? Clin Cardiol. 2020.

36. Cosentino N, Assanelli E, Merlino L, Mazza M, Bartorelli AL. An In-hospital Pathway for Acute Coronary Syndrome Patients During the COVID-19 Outbreak: initial Experience Under Real-World Suboptimal Conditions. Can J Cardiol. 2020;36 (6):961-964.

37. Birkmeyer JD, Barnato A, Birkmeyer N, Bessler R, Skinner J. The Impact Of The COVID-19 Pandemic On Hospital Admissions In The United States. Health Aff. 2020;39(11): 2010-2017.

38. Mesnier J, Cottin Y, Coste P, et al. Hospital admissions for acute myocardial infarction before and after lockdown according to regional prevalence of COVID-19 and patient profile in France: a registry study. Lancet Public Health. 2020;5(10): e536-e542.

39. De Rosa S, Spaccarotella C, Basso C, et al. Reduction of hospitalizations for myocardial infarction in Italy in the COVID-19 era. Eur Heart J. 2020;41(22):2083-2088.

40. Jimenez-Blanco Bravo M, Cordero Pereda D, Sanchez Vega D, et al. Heart Failure in the Time of COVID-19. Cardiology. 2020;145(8):481-484.

41. Almufleh A, Ahluwalia M, Givertz MM, et al. Short-term Outcomes in Ambulatory Heart Failure during the COVID-19 Pandemic: insights from Pulmonary Artery Pressure Monitoring. J Card Fail. 2020;26(7):633-634.

42. COVID-19 Pulse: delivering regular insights on the pandemic from a $150,000+$ person connected cohort. Available from: https://evidation.com/news/covid-19-pulse-first-data-evidation/. Accessed January 25, 2021.

43. Emami A, Javanmardi F, Pirbonyeh N, Akbari A. Prevalence of Underlying Diseases in Hospitalized Patients with COVID-19: a Systematic Review and Meta-Analysis. Arch Acad Emerg Med. 2020;8(1):e35.

44. Wang B, Li R, Lu Z, Huang Y. Does comorbidity increase the risk of patients with COVID-19: evidence from meta-analysis. Aging. 2020;12(7):6049-6057.

45. Singh AK, Gillies CL, Singh R, et al. Prevalence of co-morbidities and their association with mortality in patients with COVID-19: a systematic review and meta-analysis. Diabetes Obes Metab. 2020.

46. Bajgain KT, Badal S, Bajgain BB, Santana MJ. Prevalence of comorbidities among individuals with COVID-19: a rapid review of current literature. Am J Infect Control. 2020.

47. Basu S. Non-communicable disease management in vulnerable patients during Covid-19. Indian J Med Ethics. 2020;V(2): 103-105. 
48. Ibrahim JE, Anderson LJ, MacPhail A, Lovell JJ, Davis MC, Winbolt M. Chronic disease self-management support for persons with dementia, in a clinical setting. $J$ Multidiscip Healthc. 2017;10:49-58.

49. Snowden MB, Steinman LE, Bryant LL, et al. Dementia and co-occurring chronic conditions: a systematic literature review to identify what is known and where are the gaps in the evidence? Int J Geriatr Psychiatry. 2017;32(4):357-371.

50. Hariyanto TI, Putri C, Arisa J, Situmeang RFV, Kurniawan A. Dementia and outcomes from coronavirus disease 2019 (COVID-19) pneumonia: a systematic review and meta-analysis. Arch Gerontol Geriatr. 2020;93:104299.

51. Al-Quteimat OM, Amer AM. The Impact of the COVID-19 Pandemic on Cancer Patients. Am J Clin Oncol. 2020;43 (6):452-455.

52. Gupta A, Bush A, Nagakumar P. Asthma in children during the COVID-19 pandemic: lessons from lockdown and future directions for management. Lancet Respir Med. 2020;8(11):10 70-1071.

53. Simoneau T, Greco KF, Hammond A, Nelson K, Gaffin JM. Impact of the COVID-19 Pandemic on Pediatric Emergency Department Utilization for Asthma. Ann Am Thorac Soc. 2020.

54. Hall ME, Vaduganathan M, Khan MS, et al. Reductions in Heart Failure Hospitalizations During the COVID-19 Pandemic. $J$ Card Fail. 2020;26(6):462-463.

55. Virani SA, Clarke B, Ducharme A, et al. Optimizing Access to Heart Failure Care in Canada During the COVID-19 Pandemic. Can J Cardiol. 2020;36(7):1148-1151.

56. Kitsiou S, Pare G, Jaana M. Effects of home telemonitoring interventions on patients with chronic heart failure: an overview of systematic reviews. J Med Internet Res. 2015;17(3):e63.

57. Mileski M, Kruse CS, Catalani J, Haderer T. Adopting Telemedicine for the Self-Management of Hypertension: systematic Review. JMIR Med Inform. 2017;5(4):e41.

58. Ceriello A, Schnell O. COVID-19: considerations of Diabetes and Cardiovascular Disease Management. J Diabetes Sci Technol. 2020;14(4):723-724.

59. Flodgren G, Rachas A, Farmer AJ, Inzitari M, Shepperd S. Interactive telemedicine: effects on professional practice and health care outcomes. Cochrane Database Syst Rev. 2015;(9): CD002098.

60. Ghai B, Malhotra N, Bajwa SJS. Telemedicine for chronic pain management during COVID-19 pandemic. Indian J Anaesth. 2020;64(6):456-462.

61. Bokolo Anthony J. Use of telemedicine and virtual care for remote treatment in response to COVID-19 Pandemic. $J$ Med Syst. 2020;44(7):132.

62. Gao Y, Liu R, Zhou Q, et al. Application of telemedicine during the coronavirus disease epidemics: a rapid review and meta-analysis. Ann Transl Med. 2020;8(10):626.

63. Bader F, Manla Y, Atallah B, Starling RC. Heart failure and COVID-19. Heart Fail Rev. 2020

64. Elbeddini A, Tayefehchamani Y. Amid COVID-19 pandemic: challenges with access to care for COPD patients. Res Social Adm Pharm. 2020

65. Adan GH, Mitchell JW, Marson T. Epilepsy care in the COVID-19 era. Clin Med. 2020;20(4):e104-e106.

66. Brigo F, Bonavita S, Leocani L, Tedeschi G, Lavorgna L, Digital Technologies W. Social Media Study Group of the Italian Society of $\mathrm{N}$ : telemedicine and the challenge of epilepsy management at the time of COVID-19 pandemic. Epilepsy Behav. 2020;110:10 7164

67. Nouri S, Khoong EC, Lyles CR, Karliner L. Addressing equity in telemedicine for chronic disease management during the covid-19 pandemic. NEJM Catalyst Innovation in Care Delivery. 2020.
68. Eberly LA, Khatana SAM, Nathan AS, et al. Telemedicine outpatient cardiovascular care during the covid-19 pandemic: bridging or opening the digital divide? Circulation. 2020.

69. Mobile Fact Sheet. Available from: https://www.pewresearch.org/ internet/fact-sheet/mobile/. Accessed January 25, 2021.

70. Nouri SS, Avila-Garcia P, Cemballi AG, Sarkar U, Aguilera A, Lyles CR. Assessing mobile phone digital literacy and engagement in user-centered design in a diverse, safety-net population: mixed methods study. JMIR Mhealth Uhealth. 2019;7(8):e14250.

71. Badreldin HA, Atallah B. Global drug shortages due to COVID-19: impact on patient care and mitigation strategies. Res Social Adm Pharm. 2020.

72. Liu S, Luo P, Tang M, et al. Providing pharmacy services during the coronavirus pandemic. Int J Clin Pharm. 2020;42(2): 299-304.

73. Kanji S, Burry L, Williamson D, et al. Therapeutic alternatives and strategies for drug conservation in the intensive care unit during times of drug shortage: a report of the Ontario COVID-19 ICU Drug Task Force. Can J Anaesth. 2020;67 (10):1405-1416.

74. Choo EK, Rajkumar SV. Medication shortages during the COVID-19 Crisis: what We Must Do. Mayo Clin Proc. 2020;95 (6):1112-1115.

75. Romano S, Galante H, Figueira D, Mendes Z, Rodrigues AT. Time-trend analysis of medicine sales and shortages during COVID-19 outbreak: data from community pharmacies. Res Social Adm Pharm. 2020.

76. Cundell T, Guilfoyle D, Kreil TR, Sawant A. Controls to minimize disruption of the pharmaceutical supply chain during the COVID-19 pandemic. PDA J Pharm Sci Technol. 2020;74 (4):468-494.

77. Attaway A, Hatipoglu U. Management of patients with COPD during the COVID-19 pandemic. Cleve Clin J Med. 2020.

78. Lopez-Sanchez GF, Lopez-Bueno R, Gil-Salmeron A, et al. Comparison of physical activity levels in Spanish adults with chronic conditions before and during COVID-19 quarantine. Eur J Public Health. 2020.

79. Hudson GM, Sprow K. Promoting physical activity during the covid-19 pandemic: implications for obesity and chronic disease management. $J$ Phys Act Health. 2020;1-3.

80. Yang YC, Chou CL, Kao CL. Exercise, nutrition, and medication considerations in the light of the COVID pandemic, with specific focus on geriatric population: a literature review. $J$ Chin Med Assoc. 2020;83(11):977-980.

81. Assaloni R, Pellino VC, Puci MV, et al. Coronavirus disease (Covid-19): how does the exercise practice in active people with type 1 diabetes change? A preliminary survey. Diabetes Res Clin Pract. 2020;166:108297.

82. Mattioli AV, Sciomer S, Cocchi C, Maffei S, Gallina S. Quarantine during COVID-19 outbreak: changes in diet and physical activity increase the risk of cardiovascular disease. Nutr Metab Cardiovasc Dis. 2020;30(9):1409-1417.

83. Ong JL, Lau T, Massar SAA, et al. COVID-19 Related Mobility Reduction: heterogenous Effects on Sleep and Physical Activity Rhythms. Sleep. 2020.

84. Galle F, Sabella EA, Ferracuti S, et al. Sedentary behaviors and physical activity of italian undergraduate students during lockdown at the time of covid-19 pandemic. Int J Environ Res Public Health. 2020;17:17.

85. Caci G, Albini A, Malerba M, Noonan DM, Pochetti P. COVID19 and Obesity: dangerous Liaisons. J Clin Med. 2020;9:8.

86. da Silveira MP, da Silva Fagundes KK, Bizuti MR, Starck E, Rossi RC, Resende ESDT. Physical exercise as a tool to help the immune system against COVID-19: an integrative review of the current literature. Clin Exp Med. 2020. 
87. DeJean D, Giacomini M, Vanstone M, Brundisini F. Patient experiences of depression and anxiety with chronic disease: a systematic review and qualitative meta-synthesis. Ont Health Technol Assess Ser. 2013;13(16):1-33.

88. Shanthanna H, Strand NH, Provenzano DA, et al. Caring for patients with pain during the COVID-19 pandemic: consensus recommendations from an international expert panel. Anaesthesia. 2020;75(7):935-944.

89. Black PH, Garbutt LD. Stress, inflammation and cardiovascular disease. J Psychosom Res. 2002;52(1):1-23.

90. Lovallo WR, Gerin W. Psychophysiological reactivity: mechanisms and pathways to cardiovascular disease. Psychosom Med. 2003;65(1):36-45.

91. Steptoe A, Kivimaki M. Stress and cardiovascular disease. Nat Rev Cardiol. 2012;9(6):360-370.

92. Umucu E, Lee B. Examining the impact of COVID-19 on stress and coping strategies in individuals with disabilities and chronic conditions. Rehabil Psychol. 2020;65(3):193-198.

93. Kuroda N. Mental health considerations for patients with epilepsy during COVID-19 crisis. Epilepsy Behav. 2020;111:107198.

94. Hao X, Zhou D, Li Z, et al. Severe psychological distress among patients with epilepsy during the COVID-19 outbreak in southwest China. Epilepsia. 2020;61(6):1166-1173.

95. Miller WR, Von Gaudecker J, Tanner A, Buelow JM. Epilepsy self-management during a pandemic: experiences of people with epilepsy. Epilepsy Behav. 2020;111:107238.

96. Fu W, Wang C, Zou L, et al. Psychological health, sleep quality, and coping styles to stress facing the COVID-19 in Wuhan, China. Transl Psychiatry. 2020;10(1):225.
97. Robillard R, Dion K, Pennestri M, et al. Profiles of sleep changes during the COVID-19 pandemic: demographic, behavioral and psychological factors. J Sleep Res. 2020.

98. Robillard R, Dion K, Pennestri MH, et al. Profiles of sleep changes during the COVID-19 pandemic: demographic, behavioural and psychological factors. J Sleep Res. 2020;e13231.

99. Li Y, Qin Q, Sun Q, Sanford LD, Vgontzas AN, Tang X. Insomnia and psychological reactions during the COVID-19 outbreak in China. J Clin Sleep Med. 2020;16(8):1417-1418.

100. Gualano MR, Lo Moro G, Voglino G, Bert F, Siliquini R. Effects of Covid-19 lockdown on mental health and sleep disturbances in italy. Int J Environ Res Public Health. 2020;17:13.

101. Simpson N, Manber R. Treating insomnia during the COVID-19 pandemic: observations and perspectives from a behavioral sleep medicine clinic. Behav Sleep Med. 2020;18(4):573-575.

102. Voitsidis P, Gliatas I, Bairachtari V, et al. Insomnia during the COVID-19 pandemic in a Greek population. Psychiatry Res. 2020;289:113076.

103. Morin CM, Carrier J. The acute effects of the COVID-19 pandemic on insomnia and psychological symptoms. Sleep Med. 2020.

104. Voulgaris A, Ferini-Strambi L, Steiropoulos P. Sleep medicine and COVID-19. Has a New Era Begun? Sleep Med. 2020; 73:170-176

105. Del Campo F, Lopez G, Arroyo CA, et al. Study of the adherence to continuous positive airway pressure treatment in patients with obstructive sleep apnea syndrome in the confinement during the COVID-19 pandemic. Arch Bronconeumol. 2020.
Risk Management and Healthcare Policy

\section{Publish your work in this journal}

Risk Management and Healthcare Policy is an international, peerreviewed, open access journal focusing on all aspects of public health, policy, and preventative measures to promote good health and improve morbidity and mortality in the population. The journal welcomes submitted papers covering original research, basic science, clinical \& epidemiological studies, reviews and evaluations,

\section{Dovepress}

guidelines, expert opinion and commentary, case reports and extended reports. The manuscript management system is completely online and includes a very quick and fair peer-review system, which is all easy to use. Visit http://www.dovepress.com/testimonials.php to read real quotes from published authors. 